\title{
Pesticide Residues Recovery studies by QuEChERS Sample Technique Using Gas Chromatography
}

\author{
C. Sivanandha Reddy and G.V. Subba Reddy* \\ JNTUA College of Engineering (Autonomous), Pulivendula, YSR (Kadapa) Dist, A.P, India 516390
}

\begin{abstract}
The modified Quick, Easy, Cheap, Effective, Rugged, and Safe (QuEChERS) sample preparation method was developed and validated for quantification of trace level of dicofol, chlordane and dieldrin pesticides in sunflower oil. The proposed method was applied for extraction of pesticides in sunflower oil and results were plotted with the help of matrix-matched calibration using relative responses versus standard solution. The quantification of pesticide residues carried out in sunflower oil by Gas Chromatography coupled with ECD detector. The method reveals that experimental results were satisfactory in respect of various analytical parameters such as linearity, recovery and precision. The limit of detection (LOD) and limit of quantification (LOQ) for target analytes were in the range of $0.001-0.011 \mathrm{mgkg}^{-1}$ and $0.002-0.030 \mathrm{mgkg}^{-1}$ respectively. The influence activated charcoal addition to the sorbents for the recovery of target analyte was also studied and presented in the results.
\end{abstract}

Key words: Modified QuChERS method, Organochlorine pesticides, Sunflower oil, Activated charcoal, Residues

The quantification of pesticides from oil matrices is a challenging analytical task because of the high triglyceride content of the samples $(100 \%)$ and the possible presence of lipophylic analytes. The most often used techniques for extraction and cleanup are liquid-liquid partitioning with acetonitrile or acetonitrile-hexane mixture, followed by a gel-permeation chromatography or a solid phase extraction (SPE) (Li et al., 2009; Muccio et al., 2006). However, in recent time's pesticide analysis, there is an increasing need for multi-residue methods, generating the development of versatile multi-analyte sample preparation methodologies.

Several multi-residue methods have been reported for the measurement of pesticides particularly in fruits and vegetables (Soler et al., 2005; Kmell et al., 2008; Zhao et al., 2004). The QuEChERS method, which stands for Quick, Easy, Cheap, Effective, Rugged, and Safe, was introduced in 2003 by Anastassiades et al., (2003). For the determination of pesticide residues in various food products. The method was mainly based on a salt-out acetonitrile extraction followed by a dispersive solid-phase extraction (d-SPE) clean-up procedure using a primarysecondary amine (PSA) sorbent and anhydrous MgSO4. The European Union Standards (EU), Food and Agriculture Organization of the United Nations (FAO) and the World Health Organization (WHO) have defined Maximum Residue Limits (MRL) in edible oils for various pesticide residues in the range of $0.002-2.0 \mathrm{mg} / \mathrm{kg}$. However, MRL values for pesticides in edible oils have not yet been formulated in several countries including India (Pragney et al., 2014). According to International Union of Pure and Applied Chemistry (IUPAC.2009) defined the recovery

*Corresponding author email: chanda.sivanandareddy@gmail.com as the proportion of the amount of analyte present in or added to the analytic portion of the test material which is extracted and presented for measurement. In the field of pesticide analysis, the recovery values are acceptable from $70-120 \%$ to routine analysis, as recommended by the Codex Aliment arius guidelines (Khay et al., 2009) as well as EU Commission guidelines. Poor recovery and high recovery values are found in some studies due to the interference of food matrix and rich fat contained samples (Argauer et al., 1997; Przybylski and Segard, 2009). The authors (Doong and Lee, 1999; Serrano R et al., 2003) reported that the matrix interference reduced by the help of clean up procedure and achieve the good recoveries even from high fat foods.

Subsequently, the QuEChERS method was modified for analysis of high oil-content matrices particularly Avocado or Olives oils (Cunha et al., 2007). However, from the literature survey, it reveals that vegetable oils (with the exception of olive oil) have not been investigated thoroughly using QuEChERS approach (Ferreret et al., 2005; Cunha et al.,2007). The evaluation of different combinations of sorbents, such as PSA, C18 and GCB, for the dispersive SPE (dSPE) step of QuEChERS procedure has been reported (Rajski et al., 2013). The aim of present study is to evaluate the analytical performance and applicability of the proposed modified QuEChERS method on sunflower oil matrices using dispersiveSPE (d-SPE) clean-up sorbent combinations, and their effects on the method performance parameters such as recovery studies and limit of detection (LOD) were evaluated critically.

However, for sunflower oil matrices containing large amounts of pigment and fats, which can interfere the analysis, wherein active charcoal could 
play an significant role in eliminating the pigments in the extract. In the present work, further an effective sample preparation of QuEChERS method was modified in the d-SPE clean up step by using the the activated charcoal to improve the recovery values.

\section{Material and Methods \\ Chemicals and reagents}

All the chemicals used in the present study is Analar grade and solvents were used under LC-MS grade \& LC grade and procured from M/SThermo Fisher Scientific (Fair Lawn, NJ). All the pesticide standards such as chlordane, dicofol, dieldrin were supplied by M/S Sigma Aldrich, Bangalore. The QuEChERS extraction kit procured from M/S Phenomenx Company, Hyderabad.

\section{Instrumentation}

The experiments were performed in the Agilent Technologies7890 model Gas Chromatography equipped with a ECD, (Column HP-5 capillary column $0.25 \mathrm{~mm}$ i.d., $30 \mathrm{~m}$ and $0.25 \mu \mathrm{m}$ phase film diameter was used. The total run time gave 40 minutes to the instrumentation. The pressure of the carrier gas (Nitrogen) at a flow of $1.0 \mathrm{~mL} \mathrm{~min}^{-1}$ was used to carry out experimentation.

\section{Extraction procedure}

The sunflower oil samples were collected from department stores from local area around Kadapa District in Andhra Pradesh, India. The modified QuEChERS procedure was adopted for extract of target analyte from the sample $3 \mathrm{~g}$ of oil sample was placed in a $50-\mathrm{mL}$ falcon tube. Subsequently, $7 \mathrm{~mL}$ of distilled water and $10 \mathrm{~mL}$ of acetonitrile solution (MeCN) were added to the tube, and it was shaken by mechanical shaker for $10 \mathrm{~min}$. QuEChERS extraction salts ( $4 \mathrm{~g}$ anhydrous $\mathrm{MgSO}_{4}, 1 \mathrm{~g} \mathrm{NaCl}$ ) were added to the tube and it was shaken again for another $10 \mathrm{~min}$ and subjected to centrifuge at 5000 rpm for $5 \mathrm{~min}$. Then, $3 \mathrm{~mL}$ of the supernatant was transferred to the dispersive centrifuge tube $(15 \mathrm{~mL})$ containing $150 \mathrm{mg}$ of PSA and $150 \mathrm{mg}$ of anhydrous $\mathrm{MgSO} 4$, added different amounts of activated charcoal(20mg,30mg,40mg and $50 \mathrm{mg}$ ) and then stirred in vortex for 2 min and centrifuged (5000 rpm for $5 \mathrm{~min}$ ). An aliquot of $2 \mathrm{~mL}$ of the $\mathrm{MeCN}$ extract was transferred to a vial, dried under a gentle nitrogen stream and the resultant residue was re-dissolved with acetonitrile solvent.

\section{Results and Discussion}

The established QuEChERS method was employed for the estimation of dicofol, chlordane and dieldrin in sunflower oil. The low volatility also one of the factor not affect quantitation. However, fat and pigments can thermally degrade in the injection linear or remain in the columns which can lead to increase maintenance of the instrument frequently. As a result, the pesticide residues analysis in oil samples such as sunflower oil, Ground nut oils, Olive oil and other edible oils could be difficult task. In an analytical method, various types of extraction methods and clean up steps are to be followed to obtain the maximum analyte recovery with minimum matrix interference at the final measurement step (Bennett et.al., 1997). Several permutation QUEChERS sample preparation approaches are useful to improve results or practical efficiency of recovery for the targeted analyte. In this study the modified QuEChERS has been adopted by employing activated charcoal and effectively extracted the title compound from samples. The accurate quantification of pesticide residues normally expressed in terms of recovery value of analyte.

The different concentrations of extractants of analytes were subjected for gas chromatography and evaluated analytical data is depicted in Table 1.

Table 1. Recovery values (\%) with applied different concentrations of AC

\begin{tabular}{cccc}
\hline $\begin{array}{c}\text { Amount of } \\
\text { activated } \\
\text { charcoal }(\mathrm{mg})\end{array}$ & Dicofol & Chlordane & Dieldrin \\
\hline 20 & 79 & 95 & 101 \\
30 & 82 & 99 & 103 \\
40 & 85 & 102 & 105 \\
50 & 80 & 94 & 95 \\
\hline
\end{tabular}

Even though the activated charcoal can remove the fat and pigments, too much of it can also remove the analytes of interest, which leads to lower recoveries. The well-defined chromatogram with

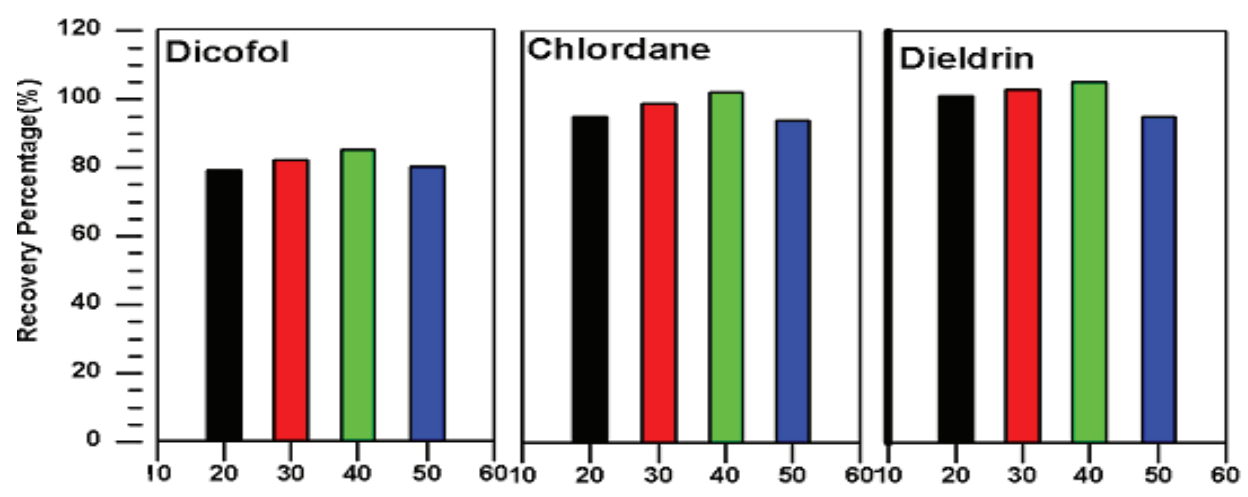

Fig. 1 Effect of AC in dSPE method on extraction recoveries in graphical representation 
minimum interference of co extractants, reveal that activated charcoal in the cleanup step played an important role in the quantification the analyte even at lower concentration. Further, the employed activated evaluated in terms of relative standard deviations (RSDs, \%) were each pesticide residues namely dicofal was $1.6 \%$, Chlordane was $3.8 \%$ and Dieldren was $2.4 \%$. The retention time was observed for

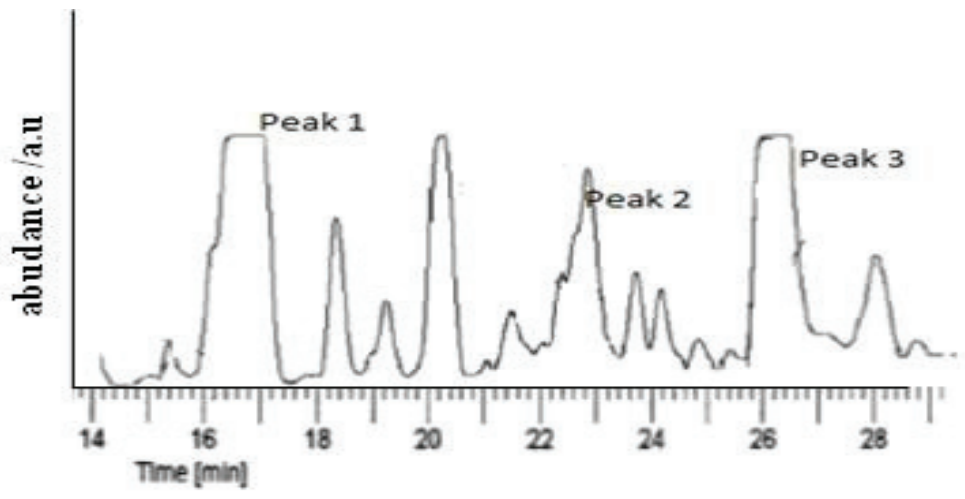

Fig. 2 GC-ECD Chromatogram of with matrix. Dicofol (Peak1), Chlordane (Peak2),

charcoal is having large surface area, could be reason for efficient adsorption, which intern eliminated the co-extractants. The recoveries of analyte by using different amount of activated charcoal have been these the three pesticides are $16,22.5$, and 26.5 minutes respectively. The validated procedure was employed for recovery studies of spiked samples by using different concentration of $A C$ and found to be

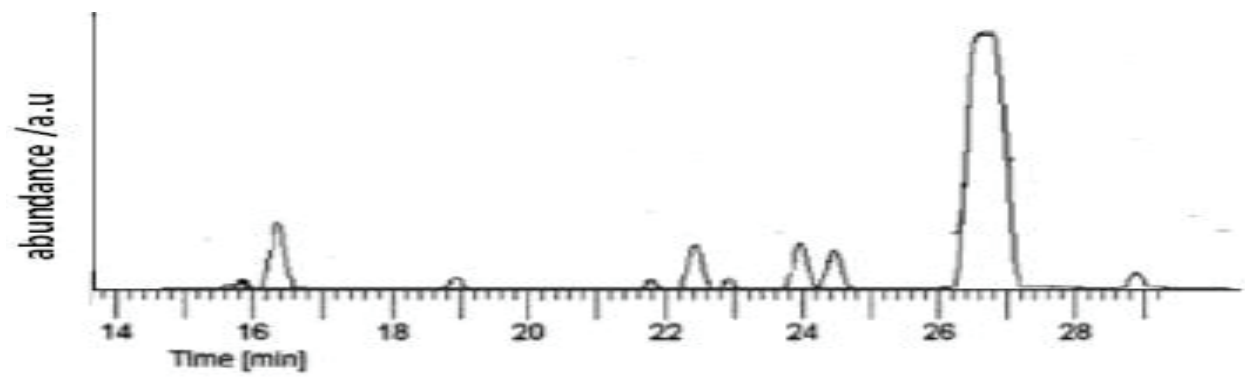

Fig. 3 GC-ECD chromatogram of standards solution

studied and evaluated quantitative data is shown in Table 1 and its graphical representation depicted in Figure 1. From the recovery studies found that best values obtained in the case of $40 \mathrm{mg} \mathrm{AC}$ used in optimized QuEChERS method.

\section{Method validation}

The analytical method validation was carried out on the basis of SANCO guidelines (E.C.2009). The LOD of the method for each pesticide was measured at signal-to-noise $(\mathrm{S} / \mathrm{N})$ ratio of 3:1. The $L O Q$ values calculated at signal-to-noise $(\mathrm{S} / \mathrm{N})$ of $10: 1$. The limit of detection (LOD) and limit of quantification (LOQ) for target analytes were in the range of $0.001-0.011$ $\mathrm{mg} \mathrm{kg}^{-1}$ and $0.002-0.030 \mathrm{mg} \mathrm{kg}^{-1}$ respectively. The linearity of the developed method was tested over the concentration range of 5 to $1000 \mathrm{ng} / \mathrm{mL}$ by spiked the each pesticide standard sample. The regression coefficient (R2) values calculated for Dicofal, Chlordane, Dieldrin and found to 0.992, 0.995, and 0.998 respectively. The calculated precision values in the range of $79 \%-105 \%$ and results were shown in Table 1 and Fig. 1.

\section{Conclusion}

The modified and established QuEChERS method for the extraction of organochlorine pesticides in samples of sunflower oils was employed and subsequent quantification by GC-ECD showed good precision, accuracy, linearity and sensitivity. Hence, it recommended for the routine analysis of various pesticide samples. The extracted analytes were clearly detected without interference from matrix in the proportional addition of AC to PSA sorbent. The clean - up efficiency also improved by proposed method. The validated method proved to be a fast and effective. In the modified QuChERES method combinations are PSA+AC economically very cheap as compare with PSA and GCB sorbent combinations. The optimum quantity of $A C$ proposed for good analyte recoveries but higher quantity of $A C$ causes lower recoveries. 


\section{Acknowledgment}

The authors are grateful to the Technical Education Quality Improvement Program (TEQIP-II) for financial support to carry out the research project.

\section{References}

Anastassiades, M., Lehotay, S.J., Stajnbaher, D. and Schenck, F.J. 2003. Fast and easy multi residue method employing acetonitrile extraction/partitioning and dispersive SPE for the determination of pesticide residues in produce. J. AOAC Int., 86: 412- 431.

Argauer, J.R., Lehotay, J.S. and Brown, T.R. 1997. Determining Lipophilic Pyrethroids and Chlorinated Hydrocarbons in Fortified Ground Beef Using IonTrap Mass Spectrometry", J. Food Chem., 45(10): 3936-3939.

Bennett, A.D., Chung, C.A. and Lee, M.S. 1997. Multiresidue method for analysis of pesticides in liquid whole milk. J. AOAC Int., 80:1065-77.

Cunha, C.S., Fernandes, O.J. and Oliveira, M. 2007. Comparison of matrix solid-phase dispersion and liquid-liquid extraction for the chromatographic determination of fenthion and its metabolites in olives and olive oils. Food Addit. Contam., 24: 156-164.

Cunha, C.S., Lehotay, J.S. Mastovska, K., Fernandes, O.J., Beatriz, M. and Oliveira, P.P. 2007. Evaluation of the QUECHERS sample preparation approach for the analysis of pesticide residues in olives. J. Sep Sci., 30: 620-632.

Doong, R.A. and Lee, C.Y. 1999. Determination of organochlorine pesticide residues in foods using solid-phase extraction clean-up cartridges. Analyst ., 124(9):1287-9.

EC. 2009. Method validation and quality control procedures for pesticide residues analysis in food and feed. Document SANCO/10684/2009. [http://ec.europa.eu/ food/plant--/protection/resources/qualcontrol_en.pdf and http://www.eurlpesticides.eu/docs/--public/ tmplt_article.asp?CntID=727\&LabID=100\&Lang=EN].

Ferrer, C., Omez, G.J.M., Garcia-Reyes, I., Ferrer,J.F., Thurman, M.E. and Ernandez-Alba,R.A., 2005. A Determination of pesticide residues in olives and olive oil by matrix solid-phase dispersion followed by gas chromatography/mass spectrometry and liquid chromatography/tandem mass spectrometry. J. Chromatog. A.,1069:183-194.

IUPAC.,1996. Symposium on harmonisation of quality assurance systems for analytical laboratories, 4-5 September 1996, Orlando.

Kmellar, B., Fodor, P., Pareja, L., Ferrer, C., Mart_inez-Uroz, A.M., Valverde, A. and Fernandez-Alba, R. A. 2008.
Validation and uncertainty study of a comprehensive list of 160 pesticide residues in multi-class vegetables by liquid chromatography-tandem mass spectrometry. J. Chromatogr. A., 1215: 37-50.

Khay,S., Abd El-Aty, A.M., Choi, J.H., Shin, H.J., Shin, C.H., Kim, S.J., Chang B.J,. Lee, H.C., Shin, H.S., Jeong, J.Y. and Shim. 2009. Simultaneous determination of pyrethroids from pesticide residues in porcine muscle and pasteurized milk using GC. J. Sep.Sci., 32(2): 244-251.

Li, L. W., Qin, D.,Jiang, S. and Liu, F. 2009. Application of graphitized carbon blackto the QuEChERS method for pesticide multiresidue analysis in spinach. J. AOAC Int., 92(2): 538-547.

Muccio, Di.A., Fidente, P., Barbini, A.D., Dommarco,R,. Seccia,S, and Morrica., 2006. Application of solidphase extraction and liquid chromatography-mass spectrometry to the determination of neonicotinoid pesticide residues in fruit and vegetables. J.Chromatog. A., $13: 1108,1-6$.

Pragney, D., Azmeera,T., Prabhavathi Devi, B.L.A., Padmaja, R., Prasad, R.B.N. and Vijaya Sarathi, R.V.U. 2014 .An improved dispersive solid-phase extraction cleanup method for the gas chromatography-negative chemical ionisation tandem mass spectrometric determination of multiclass pesticide residues in edible oils. J.Food Chem., $142: 144-151$.

Przybylski, C. and Segard, C. 2009. Method for routine screening of pesticides and metabolites in meat based baby-food using extraction and gas chromatographymass spectrometry. J. Sep. Sci., 32(11): 1858-67.

Rajski, L., Lozano,A., Uclés,A., Ferrer, C., and FernándezAlba, A.R., 2013. Determination of pesticide residues in high oil vegetal commodities by using various multi-residue methods and clean-ups followed by liquid chromatography tandem mass spectrometry., J. Chromatogr.A, 13(4): 109-120.

Serrano, R. Barreda, M. Pitarch,E. and Hernandez,F.J.2013. Determination of low concentrations of organochlorine pesticides and PCBs in fish feed and fish tissues from aquaculture activities by gas chromatography with tandem mass spectrometry. Sep. Sci., 26: 75-86.

Soler, C., Manes, J., and Pico, Y., 2005. Routine application using single quadrupole liquid chromatography-mass spectrometry to pesticides analysis in citrus fruits. J.Chromatogr, A., 10: 224-233.

Zhao, G., Wang, C., Wu, Q. and Wang,Z., 2011.Determination of carbamate pesticides in water and fruit samples using carbon nanotube reinforced hollow fiber liquid -phase micro extraction followed by high performance liquid chromatography, Analy. Methods., 3:1410-1417 